\title{
Evaluation of atherosclerotic findings in patients with nonalcoholic fatty liver disease
}

This article was published in the following Dove Press journal:

International Journal of General Medicine

2I October 201 I

Number of times this article has been viewed

\author{
Afshin Mohammadi' \\ Ali Bazazi ${ }^{2}$ \\ Mohammad Ghasemi-rad ${ }^{2}$ \\ 'Department of Radiology, Urmia \\ University of Medical Sciences, \\ Urmia, West-Azerbaijan, Iran; \\ ${ }^{2}$ Student Research Committee, \\ Urmia University of Medical Sciences, \\ Urmia, West-Azerbaijan, Iran
}

Background: Nonalcoholic fatty liver disease (NAFLD), a highly prevalent condition, is a feature of metabolic syndrome and is characterized by excessive accumulation of fat in the liver cells. The purpose of this study was to investigate the association between NAFLD and carotid intima-media thickness (CIMT) as an independent risk factor for atherosclerosis.

Methods: We examined 250 consecutive patients with ultrasonographically confirmed NAFLD and 85 age-matched and gender-matched controls with normal parenchymal liver echogenicity for determination of CIMT and presence of carotid atherosclerotic plaque.

Results: Compared with control subjects, patients with NAFLD showed an increased mean CIMT $(0.81 \pm 0.14 \mathrm{~mm})$ compared with control subjects $(0.58 \pm 0.15 \mathrm{~mm})$. This difference was statistically significant $(P=0.001)$. After performing multivariate analysis, the presence of NAFLD was associated with abnormal CIMT independent of other atherogenic risk factors or its association with metabolic syndrome.

Conclusion: NAFLD can be an independent risk factor for developing atherosclerosis. Therefore, NAFLD with and without metabolic syndrome can be associated with increased CIMT and increased risk of cardiovascular events in patients with NAFLD incidentally diagnosed on abdominal ultrasonography.

Keywords: nonalcoholic fatty liver disease, metabolic syndrome, atherosclerosis, carotid intima media thickness, risk factor

\section{Introduction}

Nonalcoholic fatty liver disease (NAFLD), a highly prevalent condition, ${ }^{1}$ is a feature of metabolic syndrome and characterized by excessive accumulation of fat in the liver cells. ${ }^{2,3}$ Patients with NAFLD have a higher mortality rate than the general population. ${ }^{4}$

NAFLD is strongly associated with cardiovascular risk factors, such as obesity, dyslipidemia, type 2 diabetes mellitus, and insulin resistance. ${ }^{5}$ From previous published data, it is obvious that coronary heart disease mortality rates in patients with NAFLD are close to those associated with cirrhosis. ${ }^{6}$

Ultrasonographic measurement of carotid intima-media thickness (CIMT) is a noninvasive method for demonstrating subclinical atherosclerosis. Increased CIMT has a significant relationship with the presence and severity of coronary atherosclerosis. ${ }^{7,8}$

In recent years, case-control studies have shown a relationship between NAFLD and the presence of early manifestations of atherosclerosis indicated by CIMT measurement. ${ }^{8,9}$ Other studies have shown only a relationship between NAFLD and advanced atherosclerosis, such as carotid plaque. ${ }^{10,11}$ However, the association between
Correspondence: Mohammadi Afshin Urmia University of Medical Sciences, Urmia, West-Azerbaijan, Iran

Tel +98 44| $235356 \mid$

Fax +98 44I 2353561

Emailmohamadi_afshin@yahoo.com 
CIMT and NAFLD requires further investigation because metabolic syndrome comprises multiple risk factors for coronary artery disease, which may have confounded the results of studies published to date.

In the present study, we investigated the association between NAFLD as a risk factor for atherosclerosis and CIMT by comparing a group of outpatients with NAFLD confirmed incidentally on ultrasonography and a control group with ultrasonographically normal liver echogenicity. Independent evaluation of CIMT in patients with NAFLD and other cardiovascular risk factors is the novelty of our present research.

\section{Materials and methods}

We examined 250 consecutive patients with incidental ultrasonographically confirmed NAFLD and 85 age-matched and gender-matched control subjects with normal parenchymal liver echogenicity for determination of CIMT and presence of carotid atherosclerotic plaque. The patients were referred to the Radiology Department from the Gastroenterology Outpatient Clinic at the University Training Hospital for measurement of CIMT after abdominal ultrasonography was performed for nonspecific abdominal discomfort, and diagnosis of fatty liver was made according to ultrasonographic examination. All of the abdominal sonograms were performed by one radiologist $(\mathrm{AH})$. Thirty-five patients with NAFLD were excluded from the study because they failed to meet the inclusion criteria. Exclusion criteria were heart disease, diabetes mellitus, acute or chronic liver disease, acute or chronic kidney disease, any malignancy, alcohol consumption, pregnancy, liver masses, abnormal copper metabolism or thyroid function test, history of any medication with adverse effects on the liver, and a history of cigarette smoking. Only hepatitis B surface antigen-negative and hepatitis C antibodynegative patients were enrolled.

Hypertension was diagnosed as blood pressure $>140 / 90 \mathrm{mmHg}$ or the taking of antihypertensive drugs. Body mass index was calculated by dividing weight in kilograms by height in meters squared. Blood samples were collected in the morning after an overnight fast. Blood was tested for fasting glucose (normal range $<126 \mathrm{mg} / \mathrm{dL}$ ), triglycerides (normal range $<200 \mathrm{mg} / \mathrm{dL}$ ), total cholesterol (normal range $<240 \mathrm{mg} / \mathrm{dL}$ ), high-density lipoproteins, C-reactive protein, mean platelet volume, and serum transaminases (alanine aminotransferase and aspartate aminotransferase). All blood samples were tested in the same laboratory.

High resolution $\mathrm{B}$ mode ultrasonography of both the common and internal carotid arteries was performed using an ultrasound machine (Sonoline G40; Siemens, Munich, Germany) equipped with a $10 \mathrm{mHz}$ linear array transducer. Patients were examined in the supine position with the head tilted backwards. After the carotid arteries were located by transverse scans, the probe was rotated to $90^{\circ}$ to obtain and record a longitudinal image of the common carotid arteries.

The maximum CIMT was measured at the posterior wall of the common carotid artery, $2 \mathrm{~cm}$ before the bifurcation, as the distance between the first and second echogenic lines of the anterior and posterior arterial walls. The image was focused on the posterior wall of the common carotid artery, and gain settings were used to optimize image quality. Measurement was performed vertical to the arterial wall for accurate measurement of CIMT (Figure 1). Three CIMT measurements were taken at each site and the average measurement was used. The CIMT was assessed as being normal if it did not exceed $0.7 \mathrm{~mm} .^{7}$ All of the CIMT measurement sonograms were obtained by another radiologist blinded to the results of abdominal sonography and the clinical and laboratory data for cases and control subjects.

The anterior and posterior walls of the common carotid arteries, internal carotid arteries, and carotid bulbs were evaluated for determination of presence of carotid plaque, defined as a focal thickening $>1.2 \mathrm{~mm}$ of the intima-media complex, measured from the media-adventitia interface to the intima-lumen interface (Figure 2). One radiologist experienced in Doppler evaluation of the extracranial vessels, who had no prior knowledge of the patient clinical and laboratory data, performed the ultrasonographic examinations.

Abdominal ultrasound scanning was performed in all participants by another trained radiologist, who was blinded to all patient clinical and laboratory data, using a Toshiba Nemio 30 scanner (Toshiba Co Ltd, Tokyo, Japan) with a $3.5 \mathrm{mHz}$ linear transducer. Hepatic steatosis was diagnosed by a characteristic liver echo pattern as evidence of diffuse hyperechogenicity of the liver relative to the right kidney. The patients were divided into three groups based on the degree of fatty infiltration, ie, mild fatty liver (grade 1), moderate fatty liver (grade 2), and severe fatty liver (grade 3 ) according to the ultrasound appearance of hepatosteatosis. In grade 1 (mild) fatty infiltration, echogenicity was slightly increased, with normal visualization of the diaphragm and the intrahepatic vessel borders. Grade 2 (moderate) fatty infiltration was established when echogenicity was moderately increased, with slightly impaired visualization of the diaphragm or intrahepatic vessels. In grade 3 (severe) fatty infiltration, echogenicity was markedly increased with poor 
visualization of the diaphragm, intrahepatic vessels, and posterior portion of the right lobe. The study was approved by the institutional review board of the university, and all the participants provided their written informed consent.

\section{Statistical analysis}

Statistical analysis was performed using SPSS software (v 16; SPSS Inc, Chicago, IL). Statistical significance was set at a two-sided $P$ value of $\leq 0.05$. Results are reported as the mean \pm standard deviation, $\mathrm{n}(\%)$ for continuous variables, and as frequencies for categorical variables. For comparison of patient and control data, one-way analysis of variance and $t$-testing were used for quantitative variables, and the Chisquare test was used to determine the statistical significance of differences in qualitative variables. The relationship for continuous variables was examined by Pearson's correlation coefficients and categorical variables by Spearman correlation analysis. Thereafter, one-way analysis of variance was utilized to compare different variables (including intimamedia thickness, fasting blood sugar, triglycerides, total cholesterol, high-density lipoprotein, low-density lipoprotein, alanine aminotransferase, aspartate aminotransferase, mean platelet volume, and body mass index) with the different grades of fatty liver disease. Multivariate modeling was done by linear regression analysis. In multivariate analysis, the presence of NAFLD was an independent variable and regression analysis was used.

\section{Results}

We examined 250 patients with NAFLD (139 male and 111 female) and 85 controls (46 male and 39 female). Because of the study design, cases and control subjects were almost identical in terms of gender and age. The mean age of patients with NAFLD was $46.5 \pm 10.5$ years and for controls was $44.8 \pm 14.1(P=0.078)$.

Patients with NAFLD had a higher frequency of elevated blood pressure than normal subjects (40\% vs $15.2 \%)$. This difference was statistically significant $(P=0.001)$. Mean fasting blood glucose was significantly $(P=0.001)$ higher in patients with NAFLD (117.16) than in normal subjects (90.77). Levels of aspartate aminotransferase $(P=0.006)$, alanine aminotransferase, mean platelet volume, and C-reactive protein were higher in patients with NAFLD than in the control group $(P=0.001)$. In patients with NAFLD, serum triglyceride and total cholesterol levels were higher $(P=0.001)$ and high-density lipoprotein cholesterol was lower $(P=0.001)$ than in control subjects. Patients with NAFLD had a significant higher body mass index than controls. The mean body mass index in patients with NAFLD was $29.88 \pm 3.88 \mathrm{~kg} / \mathrm{m}^{2}$ and in the control group was $25.29 \pm 4.19 \mathrm{~kg} / \mathrm{m}^{2}$; this difference was statistically significant $(P=0.001)$. Patients with NAFLD had a greater mean CIMT $(0.81 \pm 0.14 \mathrm{~mm})$ than control subjects $(0.58 \pm 0.15 \mathrm{~mm})$; this difference was statistically significant $(P=0.001)$. The frequency of carotid plaque in patients with NAFLD was 1.7-fold higher than in controls $(P=0.176)$. All risk factors related to metabolic syndrome (body mass index, hypertension, glucose level, hypertriglyceridemia, and high-density lipoprotein cholesterol) were significantly greater in patients with NAFLD than in control subjects $(P=0.001)$.

Table 1 shows the main clinical, ultrasonographic, and laboratory data for the patients and control subjects. In controls, the mean value of CIMT in men was $0.56 \pm 0.14 \mathrm{~mm}$ and in women was $0.61 \pm 0.16 \mathrm{~mm}$; this difference was not statistically significant $(P=0.09)$. In patients with NAFLD, mean CIMT in men was $0.79 \pm 0.12 \mathrm{~mm}$ and in women was $0.84 \pm 0.17 \mathrm{~mm}$; this difference was statistically significant $(P=0.019)$. Furthermore, after adjustment for multiple confounding factors, such as hypertension, diabetes mellitus, hypertriglyceridemia, hypercholesterolemia, and hyperglycemia, the mean CIMT in control subjects was $0.56 \pm 0.13 \mathrm{~mm}$ and in patients with NAFLD was $0.76 \pm 0.09 \mathrm{~mm}$, which was a statistically significant difference $(P=0.001)$. The presence of NAFLD was found to be associated with abnormal CIMT independent of other atherogenic risk factors.

Table I Main clinical, ultrasonographic, and laboratory data for patients with NAFLD and control subjects

\begin{tabular}{|c|c|c|c|}
\hline Variable & NAFLD & Control & $P$ value \\
\hline Number & 250 & 85 & \\
\hline $\operatorname{Sex}(M)$ & 139 & 46 & 0.9 \\
\hline Age (years) & $46.58( \pm 10.4)$ & $44.85( \pm 14.17)$ & 0.028 \\
\hline Weight (kg) & $83.90( \pm 9.53)$ & $70.3( \pm 11.27)$ & 0.001 \\
\hline BMI $\left(\mathrm{kg} / \mathrm{m}^{2}\right)$ & $29.88( \pm 3.88)$ & $25.29( \pm 4.19)$ & 0.001 \\
\hline ALT & $30.10( \pm 33.47)$ & $19.82( \pm 11.98)$ & 0.006 \\
\hline AST & $37.23( \pm 26.64)$ & $22.29( \pm 10.73)$ & 0.001 \\
\hline TG (mg/dL) & $189.14( \pm 88.1)$ & $107.05( \pm 36.97)$ & 0.001 \\
\hline $\begin{array}{l}\text { Total cholesterol } \\
(\mathrm{mg} / \mathrm{dL})\end{array}$ & $207.7 I( \pm 49.8 I)$ & $163.83( \pm 34.50)$ & 0.001 \\
\hline HDL (mg/L) & $41.05( \pm 8.62)$ & $52.69( \pm 9.03)$ & 0.001 \\
\hline $\mathrm{MPV}(\mathrm{fL})$ & $10.51( \pm 2.86)$ & $9.10( \pm 1.12)$ & 0.001 \\
\hline $\operatorname{CRP}(+)$ & $22.4 \%$ & $3.5 \%$ & 0.001 \\
\hline HTN $(+)$ & $40.8 \%$ & $15.2 \%$ & 0.001 \\
\hline Plaque (+) & $20 \%$ & $11.7 \%$ & 0.176 \\
\hline FBS (mg/L) & $117.16( \pm 61.52)$ & $90.77( \pm 15.10)$ & 0.001 \\
\hline IMT (mm) & $0.81( \pm 0.14)$ & $0.58( \pm 0.15)$ & 0.001 \\
\hline
\end{tabular}

Abbreviations: BMI, body mass index; TG, triglycerides; HDL, high-density lipoprotein; CRP, C-reactive protein; HTN, hypertension; FBS, fasting blood glucose; IMT, intima-media thickness; MPV, mean platelet volume; ALT, alanine aminotransferase; AST, aspartate aminotransferase; NAFLD, nonalcoholic fatty liver disease. 


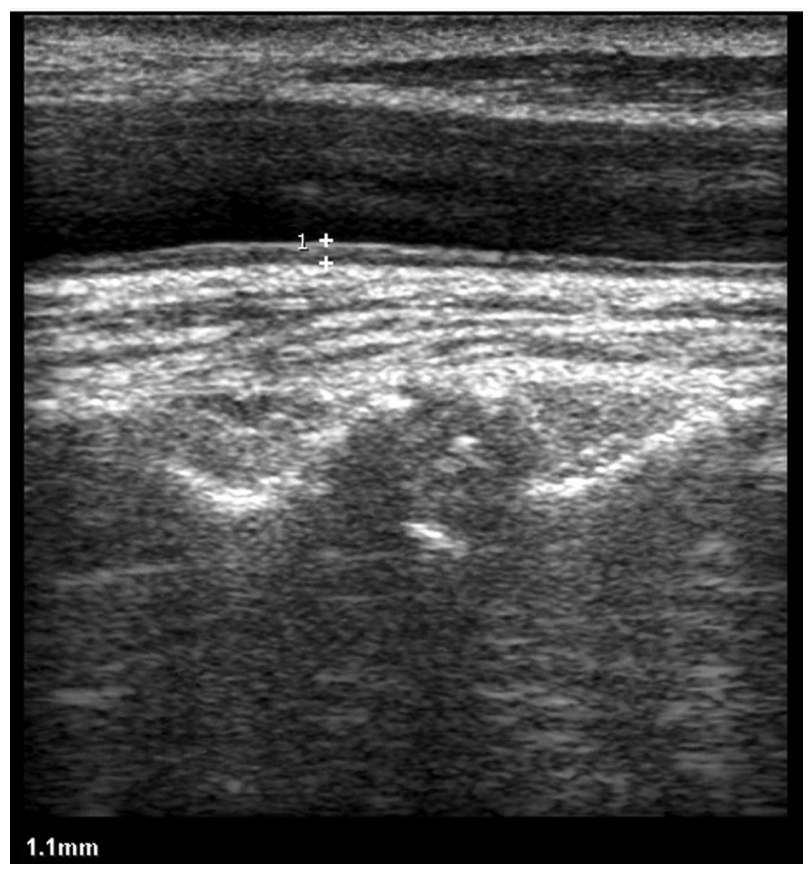

Figure I Longitudinal sonogram shows that the CIMT is $1.1 \mathrm{~mm}$ in a patient with NAFLD.

After adjustment for multiple confounding factors, mean CIMT in men with NAFLD was $0.75 \pm 0.08 \mathrm{~mm}$ and in women with NAFLD was $0.77 \pm 0.1 \mathrm{~mm}$, which was not a statistically significant difference $(P=0.36)$.

In Pearson analysis, there was a strong positive correlation between CIMT and mean platelet volume $(r=0.18, P=0.0001)$, fasting blood sugar $(r=0.18$, $P=0.0001)$, triglycerides $(r=0.18, P=0.0001)$, total cholesterol $(r=0.19, P=0.0001)$, body mass index $(r=0.39$,

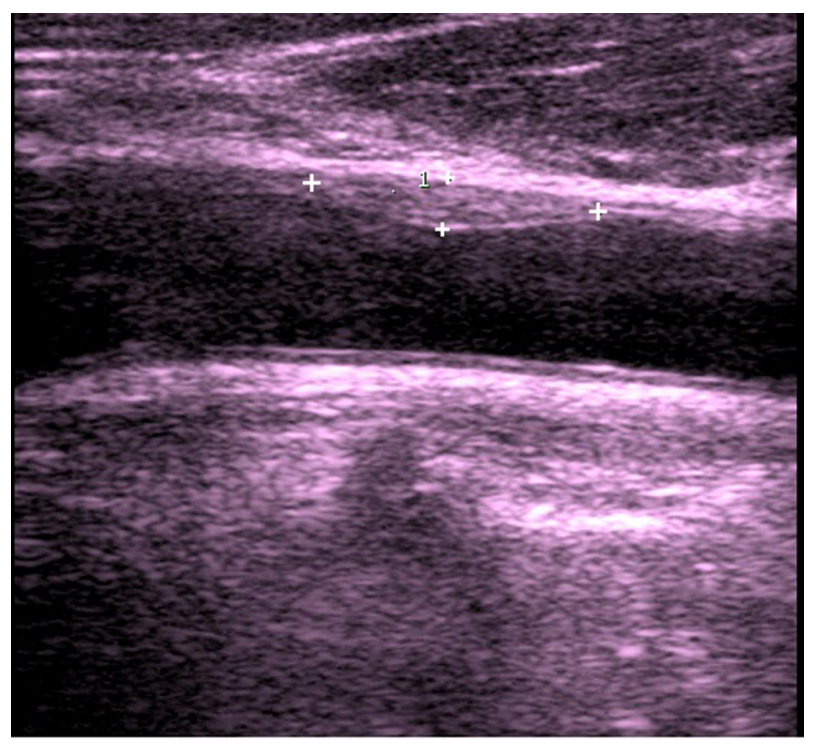

Figure 2 Longitudinal sonogram shows the presence of a soft hypoechoic carotid plaque in anterior wall of common carotid artery (CCA).
$P=0.0001)$, and age $(r=0.44, P=0.0001)$, and there was a strong negative correlation between high-density lipoprotein cholesterol and CIMT $(r=-0.32, P=0.0001)$. In multivariate linear regression analysis, the presence of elevated $C$-reactive protein $(P=0.006)$ hypertension $(P=0.005)$, and a combination of hypertension and elevated $\mathrm{C}$-reactive protein were independent risk factors affecting CIMT $(P=0.003)$.

\section{Discussion}

NAFLD is defined as excessive accumulation of fat in hepatocytes and has a range of pathologic presentations, ranging from simple steatosis to nonalcoholic steatohepatitis and cirrhosis. The prevalence of NAFLD is $20 \%-30 \%$ in the general adult population in Western countries ${ }^{12}$ and is now $12 \%-24 \%$ in Asian-Pacific countries. ${ }^{13}$ Its prevalence in obese or diabetic patients increases up to $70 \%-90 \%{ }^{12}$

Although the exact pathophysiologic basis of NAFLD is not well understood, several investigations have suggested that excessive accumulation of triglycerides in hepatocytes due to insulin resistance is the first step, and that oxidative stress reactions resulting from mitochondrial fatty acid oxidation and expression of inflammatory cytokines and adipocytokines are other potential factors which cause hepatocyte injury, inflammation, and fibrosis. ${ }^{14}$

Our study revealed that the C-reactive protein level in patients with NAFLD was higher than in control subjects $(P=0.001)$. This finding suggests that the inflammatory state caused by excessive oxidative stress reactions may contribute to hepatic injury in patients with NAFLD. The main risk factors associated with NAFLD are increased bodyweight, diabetes mellitus, hyperlipidemia, and metabolic syndrome. ${ }^{5}$ Approximately $90 \%$ of patients with NAFLD have at least one characteristic feature of metabolic syndrome and about $33 \%$ have the full complement of features. ${ }^{15,16}$ This strong association suggests that NAFLD may have a potential role in the development and progression of atherosclerosis. Some investigations suggest that the outcome in patients with NAFLD is more dependent on cardiovascular events than on the progression of liver disease. ${ }^{17-19}$

Previous investigations have demonstrated a strong relationship between CIMT and the risk of myocardial and cerebral infarction, and CIMT can identify patients at high risk for coronary artery disease effectively. ${ }^{20,21}$ Patients with a mean CIMT $>1.15 \mathrm{~mm}$ have been reported to have a $94 \%$ likelihood of having coronary artery disease. ${ }^{21}$ Many studies have evaluated the relationship between atherosclerotic risk factors and CIMT, and confirmed that CIMT thickness 
increases with age, gender, hypertension, diabetes mellitus, and hyperlipidemia. ${ }^{22,23}$

In this case-control study, we evaluated carotid atherosclerosis by measuring CIMT as an indicator of cardiovascular risk in patients with incidental ultrasonographically confirmed fatty liver. We established the diagnosis of NAFLD according to ultrasonographic appearance and exclusion of other known etiologies of chronic liver disease. The diagnosis was not confirmed by liver biopsy for ethical reasons. Ultrasonography can detect increased liver echogenicity and confirm the diagnosis of NAFLD, particularly when hepatic fat infiltration is more than $33 \% .{ }^{24}$ In this study, CIMT in patients with NAFLD was significantly higher than in a gender-matched and age-matched control group $(P=0.001)$. When compared with control subjects, women with NAFLD had a mean CIMT even higher than men with NAFLD $(P=0.019)$.

Our study found no gender-related difference in mean CIMT for patients with NAFLD and without metabolic syndrome, but the mean CIMT in female patients with NAFLD was higher than for male patients with metabolic syndrome $(P=0.019)$. This finding is similar to those of studies that showed several metabolic traits ${ }^{25}$ for the metabolic syndrome to have greater atherogenic effects on women than in men. ${ }^{26,27}$

Our study revealed an association between incidentally diagnosed NAFLD and CIMT, but no significant relationship between carotid plaque and NAFLD. Many studies have demonstrated an association between NAFLD and CIMT. ${ }^{28,29}$ Volzke et al showed that there is an independent association between hepatic steatosis and carotid plaques, but not with CIMT. ${ }^{30}$ Although in our study the body mass index in controls and patients with NAFLD was different, and previous data have shown that there is a relationship between body mass index and CIMT, ${ }^{31-33}$ our data confirm that most of the adverse effects of obesity are due to a high prevalence of hypertension and hyperglycemia, because these patients had a higher prevalence of cardiovascular risk factors and showed signs of early atherosclerosis.

We measured mean CIMT in the posterior wall of both common carotid arteries because this location is more reliable and more easily accessed compared with other carotid segments. Previous research has shown that collection of data from all carotid segments can predict cardiovascular risk more accurately than collection of data from only the common carotid..$^{25}$ Therefore, our study may have a potential limitation in estimation of the relationship between NAFLD and risk of cardiovascular events.
In conclusion, NAFLD may be an independent risk factor for developing atherosclerosis. Therefore, NAFLD without other cardiovascular risk factors can be associated with increased CIMT and increased risk of cardiovascular events in patients with NAFLD incidentally diagnosed on abdominal ultrasonography. NAFLD may serve as a trigger for assessment of silent carotid lesions and cardiovascular risk factors, and such patients should undergo further cardiovascular assessment.

\section{Disclosure}

The authors report no conflicts of interest in this work.

\section{References}

1. Bellentani S, Scaglioni F, Marino M, Bedogni G. Epidemiology of non-alcoholic fatty liver disease. Dig Dis. 2010;28(1):155-161.

2. Balmer ML, Dufour JF. Non-alcoholic steatohepatitis - from NAFLD to MAFLD. Ther Umsch. 2011;68(4):183-188.

3. Wong VW, Wong GL, Yip GW, et al. Coronary artery disease and cardiovascular outcomes in patients with non-alcoholic fatty liver disease. Gut. 2011. [Epub ahead of print.]

4. Tarquini R, Lazzeri C, Boddi M, Marra F, Abbate R, Gensini GF. Non-alcoholic fatty liver disease: a new challenge for cardiologists. G Ital Cardiol (Rome). 2010;11(9):660-669.

5. Mohamadi A, Ghasemi-Rad M, Zahedi H, Toldi G, Alinia T. Effect of severity of steatosis as assessed ultrasonographically on hepatic vascular indices in non-alcoholic fatty liver disease. Med Ultrason. 2011;13(3): 200-206.

6. Targher G, Marra F, Marchesini G. Increased risk of cardiovascular disease in nonalcoholic fatty liver disease: causal effect or epiphenomenon? Diabetologia. 2008;51(11):1947-1953.

7. Lim TK, Lim E, Dwivedi G, Kooner J, Senior R. Normal value of carotid intima-media thickness - a surrogate marker of atherosclerosis: quantitative assessment by B-mode carotid ultrasound. J Am Soc Echocardiogr. 2008;21(2):112-116.

8. Raitakari OT, Juonala M, Kähönen M, et al. Cardiovascular risk factors in childhood and carotid artery intima-media thickness in adulthood: the Cardiovascular Risk in Young Finns Study. JAMA. 2003;290(17): 2277-2283.

9. Kim JH, Kim SY, Jung ES, et al. Carotid intima-media thickness is increased not only in non-alcoholic Fatty liver disease patients but also in alcoholic Fatty liver patients. Digestion. 2011;84(2):149-155.

10. Sookoian S, Pirola CJ. Non-alcoholic fatty liver disease is strongly associated with carotid atherosclerosis: a systematic review. J Hepatol. 2008;49(4):600-607.

11. Choi SY, Kim D, Kang JH, et al. Nonalcoholic fatty liver disease as a risk factor of cardiovascular disease: relation of non-alcoholic fatty liver disease to carotid atherosclerosis. Korean J Hepatol. 2008;14(1):77-88.

12. Targher G, Day CP, Bonora E. Risk of cardiovascular disease in patients with nonalcoholic fatty liver disease. $N$ Engl J Med. 2010;363(14): 1341-1350.

13. Chitturi S, Farrell GC, Hashimoto E, Saibara T, Lau GK, Sollano JD. Non-alcoholic fatty liver disease in the Asia-Pacific region: definitions and overview of proposed guidelines. J Gastroenterol Hepatol. 2007; 22(6):778-787.

14. Duvnjak M, Lerotic I, Barsic N, Tomasic V, Virovic Jukic L, Velagic V. Pathogenesis and management issues for non-alcoholic fatty liver disease. World J Gastroenterol. 2007;13(34):4539-4550.

15. Marchesini G, Bugianesi E, Forlani G, et al. Nonalcoholic fatty liver, steatohepatitis, and the metabolic syndrome. Hepatology. 2003;37(4): 917-923. 
16. Rector RS, Thyfault JP, Wei Y, Ibdah JA. Non-alcoholic fatty liver disease and the metabolic syndrome: an update. World J Gastroenterol. 2008;14(2):185-192.

17. Marchesini G, Moscatiello S, Di Domizio S, Forlani G. Obesityassociated liver disease. J Clin Endocrinol Metab. 2008;93(Suppl 1): S74-S80.

18. de Alwis NM, Day CP. Non-alcoholic fatty liver disease: the mist gradually clears. J Hepatol. 2008;48(Suppl 1):S104-S112.

19. Nseir W, Shalata A, Marmor A, Assy N. Mechanisms Linking Nonalcoholic Fatty Liver Disease with Coronary Artery Disease. Dig Dis Sci. 2011. [Epub ahead of print.]

20. del Sol IA, Bots ML, Grobbee DE, Hofman A, Witteman JC. Carotid intima-media thickness at different sites: relation to incident myocardial infarction. The Rotterdam study. Eur Heart J. 2002;23(12):934-940.

21. Kablak-Ziembicka A, Tracz W, Przewlocki T, Pieniazek P, Sokolowski A, Konieczynska M. Association of increased carotid intima-media thickness with the extent of coronary artery disease. Heart. 2004;90(11): 1286-1290.

22. Cuspidi C, Ambrosioni E, Mancia G, Pessina AC, Trimarco B, Zanchetti A. Role of echocardiography and carotid ultrasonography in stratifying risk in patients with essential hypertension: the assessment of prognostic risk observational survey. J Hypertens. 2002;20(7): 1307-1314.

23. Sun Y, Lin CH, Lu CJ, Yip PK, Chen RC. Carotid atherosclerosis, intima media thickness and risk factors: an analysis of 1781 asymptomatic subjects in Taiwan. Atherosclerosis. 2002;164(1):89-94.

24. Bonora E, Targher G, Formentini G, Calcaterra F, Lombardi S, Marini F. The metabolic syndrome is an independent predictor of cardiovascular disease in type 2 diabetic subjects: prospective data from the Verona Diabetes Complications Study. Diabet Med. 2004;21(1):52-58.

25. Austin MA, Hokanson JE, Edwards KL. Hypertriglyceridemia as a cardiovascular risk factor. Am J Cardiol. 1998;81(4A):7B-12B.
26. Brea A, Mosquera D, Martín E, Arizti A, Cordero JL, Ros E. Nonalcoholic fatty liver disease is associated with carotid atherosclerosis: a case-control study. Arterioscler Thromb Vasc Biol. 2005;25(5): 1045-1050.

27. Hunt KJ, Resendez RG, Williams K, Haffner SM, Stern MP. National Cholesterol Education Program versus World Health Organization metabolic syndrome in relation to all-cause and cardiovascular mortality in the San Antonio Heart Study. Circulation. 2004;110(10):1251-1257.

28. O'Leary DH, Polak JF, Kronmal RA, Manolio TA, Burke GL, Wolfson SK Jr. Carotid-artery intima and media thickness as a risk factor for myocardial infarction and stroke in older adults. Cardiovascular Health Study Collaborative Research Group. $N$ Engl J Med. 1999;340(1):14-22.

29. Ramilli S, Pretolani S, Muscari A, Pacelli B, Arienti V. Carotid lesions in outpatients with nonalcoholic fatty liver disease. World J Gastroenterol. 2009; 15(38):4770-4774.

30. Volzke H, Robinson DM, Kleine V, Deutscher R, Hoffmann W, Ludemann J. Hepatic steatosis is associated with an increased risk of carotid atherosclerosis. World J Gastroenterol. 2005;11(12):1848-1853.

31. Caserta CA, Pendino GM, Alicante S, et al. Body mass index, cardiovascular risk factors, and carotid intima-media thickness in a pediatric population in southern Italy. J Pediatr Gastroenterol Nutr. 2010;51(2): 216-220.

32. Cotichini R, Zuin M, Rosmini F, Mele A, Marcucci F. Cardiovascular risk factors, nonalcoholic fatty liver disease, and carotid artery intimamedia thickness in an adolescent population in southern Italy. Am J Epidemiol. 2010;171(11):1195-1202.

33. Fahs CA, Smith DL, Horn GP, et al. Impact of excess body weight on arterial structure, function, and blood pressure in firefighters. Am J Cardiol. 2009;104(10):1441-1445.
International Journal of General Medicine

\section{Publish your work in this journal}

The International Journal of General Medicine is an international, peer-reviewed open-access journal that focuses on general and internal medicine, pathogenesis, epidemiology, diagnosis, monitoring and treatment protocols. The journal is characterized by the rapid reporting of reviews, original research and clinical studies across all disease areas.

\section{Dovepress}

A key focus is the elucidation of disease processes and management protocols resulting in improved outcomes for the patient.The manuscript management system is completely online and includes a very quick and fair peer-review system. Visit http://www.dovepress.com/ testimonials.php to read real quotes from published authors. 